\title{
Cloning of the XPD gene and its function in malignant melanoma cells
}

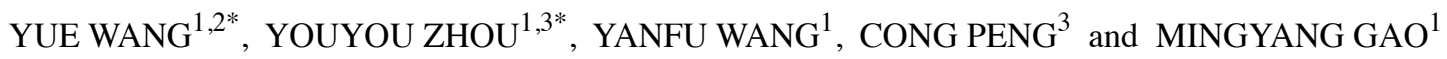 \\ ${ }^{1}$ Department of Dermatology, The First Affiliated Hospital of Dalian Medical University, Dalian, Liaoning 116011; \\ ${ }^{2}$ Department of Dermatology, Qingdao Women and Children's Hospital, Qingdao, Shandong 266000; \\ ${ }^{3}$ Department of Dermatology, Xiangya Hospital, Central South University, Changsha, Hunan 410008, P.R. China
}

Received September 25, 2019; Accepted April 3, 2020

DOI: $10.3892 / \mathrm{ol} .2020 .11708$

\begin{abstract}
The xeroderma pigmentosum group D (XPD) gene is a member of the transcription factor IIH complex and serves an important role in gene repair. Previous studies have suggested that genetic variants of the XPD gene may be associated with an increased risk of cutaneous melanoma. However, the exact mechanism remains unclear. In the present study, the XPD gene was cloned, and its localization and function in malignant melanoma cells were investigated. The human full length XPD gene was cloned via reverse transcription-PCR using the total RNA extracted from human cervical squamous cell carcinoma epithelial HeLa cells Subsequently, the gene was inserted into a plasmid fused to green fluorescent protein (GFP; pEGFP-N1/XPD), and pEGFP-N1/XPD and pcDNA3.1(+)/XPD were transfected into human malignant melanoma A375 cells using Lipofectamine ${ }^{\circledR}$ 2000. The expression levels of XPD were detected by western blotting. The Golgi marker GM130 and the endoplasmic reticulum membrane protein marker KDEL were used for immunofluorescence staining, and the subcellular localization of XPD was observed under a fluorescence microscope. Cell proliferation was measured using an MTT assay. The recombinant pEGFP-N1/XPD plasmid expressing the human wild-type XPD gene was successfully constructed by restriction enzyme digestion and assessed by gene sequencing. XPD was localized in the endoplasmic reticulum of malignant melanoma A375 cells, as confirmed by immunofluorescence staining. Furthermore, MTT assays indicated that XPD inhibited the proliferation of malignant melanoma A375 cells. The present study provides a basis for further investigation of the biological effects and functions of XPD in malignant melanoma cells.
\end{abstract}

Correspondence to: Professor Mingyang Gao, Department of Dermatology, The First Affiliated Hospital of Dalian Medical University, 222 Zhongshan Road, Dalian, Liaoning 116011, P.R. China E-mail: dlgaomingyang@126.com

${ }^{*}$ Contributed equally

Key words: XPD, clone, function, malignant melanoma

\section{Introduction}

UV radiation, smoking and various other environmental factors can cause DNA damage (1). In the human body, DNA damage can be corrected by direct repair, base excision repair and nucleotide excision repair (NER) to maintain genome stability. Among these, the NER system is the primary repair method (1). If DNA damage is not removed or repaired in time, it can lead to various diseases, such as cancer and neurodegenerative diseases, Parkinson's disease, Alzheimer's disease, Huntington's disease, aging (2). Xeroderma pigmentosum group D (XPD) is a major protein involved in NER. As an important DNA repair gene, it serves an essential role in transcription factor IIH (TFIIH) protein complex-mediated NER and its transcription process. Mutations in the human embryonic XPD gene can result in xeroderma pigmentosum, Cockayne syndrome and fur dystrophy syndrome (3). The risk of skin cancer in individuals with xeroderma pigmentosum is 1,000 times higher than that in normal individuals. From a molecular basis, xeroderma pigmentosum is a birth defect of NER in which UVB-associated DNA damage cannot be repaired (4). Previous studies have revealed associations between XPD gene polymorphisms and prostate cancer, lung cancer, bladder cancer, basal cell carcinoma and malignant melanoma (5-8). Investigations into the molecular mechanism of the susceptibility to cutaneous melanoma has revealed that mutated XPD is a biomarker of skin malignant melanoma (2). However, to the best of our knowledge, the role of the XPD gene and its protein product in malignant melanoma cells has not yet been reported.

In the present study, the XPD gene was cloned and the recombinant pEGFP-N1/XPD and pcDNA3.1(+)/XPD expression plasmids were constructed and transfected into human malignant melanoma cells. By observing XPD expression, its intracellular localization and its effects on cellular proliferation, the biological effects of XPD on malignant melanoma cells were analyzed.

\section{Materials and methods}

Reagents and antibodies. Human cervical squamous cell carcinoma epithelial HeLa cells and malignant melanoma A375 cells were purchased from The Cell Bank of Type 
Culture Collection of the Chinese Academy of Sciences. pEGFP-N1 and pcDNA3.1(+) plasmids were obtained from Clontech Laboratories, Inc. and Invitrogen (Thermo Fisher Scientific, Inc.), respectively. Restriction enzymes, RNAiso extraction reagent, reverse transcription reagent part of the PrimeScript $^{\mathrm{TM}}$ RT reagent and PrimeSTAR high fidelity enzyme were purchased from Takara Bio, Inc. Lipofectamine ${ }^{\circledR}$ 2000 was purchased from Invitrogen (Thermo Fisher Scientific, Inc.). T4 DNA ligase and the DNA gel recovery and plasmid extraction kits were obtained from Promega Corporation. The XPD antibody was ordered from Abcam (cat. no. ab102682), while GM130 (cat. no. sc-55590) and KDEL antibodies (cat. no. sc-58774) were obtained from Santa Cruz Biotechnology, Inc. Goat anti-rabbit IgG-HRP-labeled (cat. no. 4030-05), goat anti-rabbit IgG-FITC-labeled (cat. no. sc-2012) and goat anti-mouse IgG-TRITC-labeled (cat. no. 4030-03) antibodies were provided by OriGene Technologies, Inc. DMEM and FBS were purchased from Gibco (Thermo Fisher Scientific, Inc.). RIPA buffer (cat. no. P0013C) was purchased from (Beyotime, Institute of Biotechnology). Primers were synthesized by Shanghai Shenggong Biology Engineering Technology Service, Ltd., and the enhanced chemiluminescence (ECL) reagent was obtained from Merck KGaA.

Construction of the pEGFP-NI/XPD recombinant plasmid. HeLa cells were cultured in DMEM supplemented with $10 \%$ FBS and antibiotics (100 U/ml penicillin and $100 \mathrm{~g} / \mathrm{ml}$ streptomycin) at $37^{\circ} \mathrm{C}\left(5 \% \mathrm{CO}_{2}\right)$ for 2 weeks. Total RNA was extracted using the RNAiso reagent and reverse transcribed at $37^{\circ} \mathrm{C}$ for 15 min into cDNA. The primers were designed according to the human XPD intact mRNA sequence published on GenBank (NM_001130867) (9), and the restriction endonuclease sites of HindIII and SalI were added to the 5'-ends of the forward and reverse primers, respectively. The primer sequences were as follows: External nest primer XPD2-F1, 5'-TCAACGTGG ACGGGCTCCTGGTCTA-3' and XPD2-R1, 5'-TATTTGGCT GCATCTTTGCTACTGG-3'; nested primer XPD2-F2, 5'-CCC AAGCTTATGCGGGAGCTCAAACGCACGCTG-3' (HindIII restriction site underlined) and XPD2-R2, 5'-GCGTCGACT GGGGATGAGATCTTTTTTGGTTCCTG-3' (SalI restriction site underlined). The reaction system of the PrimeSTAR enzyme was configured according to the manufacturer's protocol, and the target gene was amplified by nested PCR using the thermocycling conditions of one cycle at $55^{\circ} \mathrm{C}$ for $15 \mathrm{sec}$ followed by 38 cycles at $72^{\circ} \mathrm{C}$ for $90 \mathrm{sec}$. The pEGFP-N1 empty vector and the purified PCR product were double-digested with HindIII and SalI sequentially. After purification and recovery of the digestion products by DNA gel recovery kit, the XPD gene fragment and the pEGFP-N1 vector fragment were ligated at a ratio of 5:1 in the presence of T4 DNA ligase, at $16^{\circ} \mathrm{C}$ for $12 \mathrm{~h}$. The ligation product was transformed into competent Escherichia coli DH5 a cells (cat. no. RR420A; Takara Bio Inc.) and cultured overnight on LB solid medium containing a final concentration of $30 \mu \mathrm{g} / \mathrm{ml}$ kanamycin. The resulting colonies were individually selected, added to Luria-Bertani medium (cat. no. CM0007; Beijing Leagene Biotech Co., Ltd.) and shaken at $37^{\circ} \mathrm{C}$ for $16 \mathrm{~h}$; the recombinant plasmid was extracted using a plasmid extraction kit and identified by $B g l I I$ digestion. The positive plasmid was sequenced by Shanghai Shenggong Biology Engineering Technology Service, Ltd.
Liposome-mediated transfection. The constructed recombinant $\mathrm{pEGFP-N1/XPD}$ expression vector was validated by DNA sequencing and subsequently transfected $(1 \mu \mathrm{g} / \mathrm{ml})$ into malignant melanoma $\mathrm{A} 375$ cells which were cultured in DMEM supplemented with $10 \% \mathrm{FBS}$ at $37^{\circ} \mathrm{C}$ in a $5 \% \mathrm{CO}_{2}$ incubator) using Lipofectamine ${ }^{\circledR} 2000$.

Western blot analysis. Total protein of the A375 cells was extracted using the RIPA buffer. The protein content was measured using the bicinchoninic acid assay (Beyotime Institute of Biotechnology). Equal amounts of protein (40 $\mu \mathrm{g})$ were separated via $12 \%$ SDS-PAGE, and the separated proteins were subsequently transferred onto a nitrocellulose membrane. Membranes were blocked with 5\% milk in TBST. Next, the membrane was incubated with the XPD (dilution, 1:500; cat. no. ab102682; Abcam) and GAPDH (dilution, 1:3,000; cat. no. 60004-1-Ig; Proteintech Group, Inc.) primary antibodies and shaken for $2 \mathrm{~h}$ at room temperature. After washing 3 times with TBST (TBS containing 0.1\% Tween-20), the membrane was incubated with a horse radish peroxidase goat anti-rabbit ( $\mathrm{IgG})$ secondary antibody (1:10,000; cat. no. ab6721; Abcam) and shaken for $2 \mathrm{~h}$ at room temperature. After washing 3 times with TBST, protein bands were visualized using an ECL reagent. GAPDH was used as the protein loading control. The experiment was repeated three times.

Immunofluorescence staining. The XPD2-F2 and XPD2-stop-R2 primers (5'-GCGTCGACTTAGGGATGA GATCTTTTTTGGTTC-3'; underlined for SalI restriction site) were used To observe the localization of the XPD gene in A375 cells. The eukaryotic expression plasmid pcDNA3.1(+)/XPD was constructed by amplification, digestion, ligation and transformation, as aforementioned, and subsequently transfected $(1 \mu \mathrm{g} / \mathrm{ml})$ into A375 cells for $48 \mathrm{~h}$ using Lipofectamine ${ }^{\circledR}$ 2000. The Golgi membrane protein marker GM130 and the endoplasmic reticulum membrane protein marker KDEL were used for labeling, and the location of the gene was detected by immunofluorescence staining. Briefly, cells were fixed with freshly prepared $4 \%$ paraformaldehyde for $10 \mathrm{~min}$ at room temperature. The cells were washed with PBS thrice for $5 \mathrm{~min}$ each time and then permeabilized with $0.2-0.5 \%$ Triton-100 (prepared in PBS) for $10 \mathrm{~min}$ at room temperature. Next, the cells were washed again thrice in PBS and subsequently blocked with $2 \% \mathrm{BSA}$ for $30 \mathrm{~min}$ at room temperature. Following blocking the cells were incubated with primary antibodies against GM130 (1:200) and KDEL (1:200) for $1 \mathrm{~h}$ at room temperature. The cells were again washed thrice with PBS and then incubated with secondary antibody (1:500) for $1 \mathrm{~h}$ at room temperature. Cells were visualized using a fluorescence microscope (magnification, $\mathrm{x} 400$ ).

MTT assay. Following pcDNA3.1(+) and pcDNA3.1(+)/XPD transfection into A375 cells for 24,48 and $72 \mathrm{~h}, 5 \times 10^{3}$ cells/well were seeded into 96-well plates. After 3 days of cell culture, $20 \mu 1 \mathrm{MTT}(5 \mathrm{mg} / \mathrm{ml})$ was added to each well for $4 \mathrm{~h}$. The culture supernatant was subsequently discarded, and $150 \mu \mathrm{l}$ triple solution of dimethyl sulfoxide was added to each well and shaken for $10 \mathrm{~min}$ to fully dissolve the purple formazan crystals. The light absorption value of each well was measured at a wavelength of $490 \mathrm{~nm}$. 
A

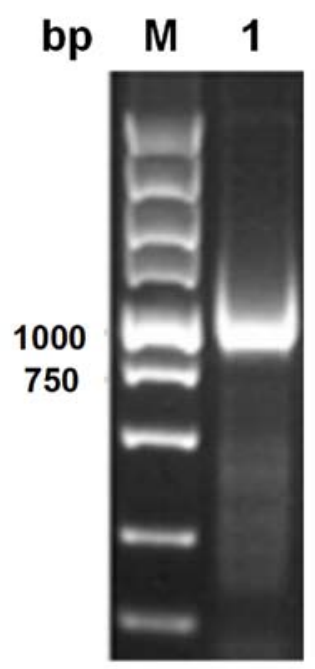

C

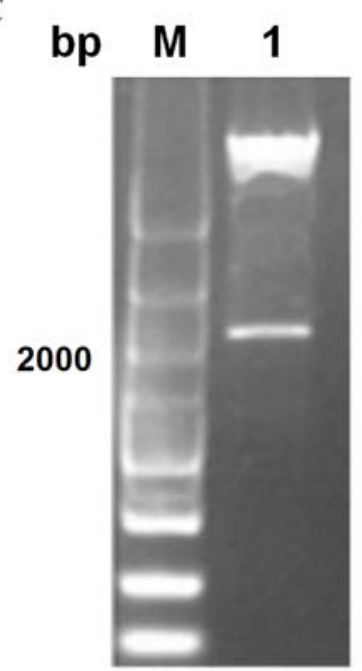

B

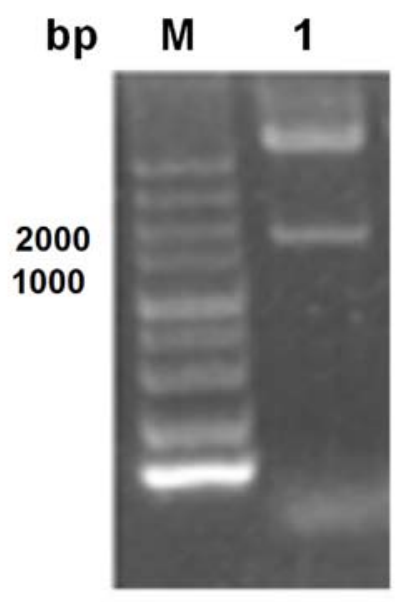

Figure 1. Cloning and identification of the XPD gene. (A) Following reverse transcription from total RNA extracted from HeLa cells, nested PCR was used to obtain a 1,234 bp product (M: DNA ladder; 1: HeLa cells PCR product). (B) BglII single digestion resulted in a 4,737 and 1,200 bp product (M: DNA ladder; 1: BgIII single digestion product). (C) pcDNA3.1(+)/XPD was obtained and identified by HindIII and XbaI digestion. XPD, xeroderma pigmentosum group D. (M: DNA ladder; 1: HindIII and SalI digestion products).

Statistical analysis. Each experiment was repeated three times and the data are presented as the mean \pm SD. Statistical analyses were conducted using SPSS v17.0 (SPSS, Inc.). A Student's unpaired t-test was used to compare differences between two groups, such as pcDNA3.1(+) $48 \mathrm{~h}$ and pcDNA3.1(+)/XPD $48 \mathrm{~h}$ groups, pcDNA3.1(+) $72 \mathrm{~h}$ and pcDNA3.1(+)/XPD $72 \mathrm{~h}$ groups. One-way ANOVA was used to compare differences among multiple groups, such as the DMEM, pcDNA3.1(+) and pcDNA3.1(+)/XPD groups. ANOVA was followed by Tukey's post hoc test. $\mathrm{P}<0.05$ was considered to indicate a statistically significant difference.

\section{Results}

Cloning and identification of the XPD gene. Following the reverse transcription of HeLa cell total RNA, a nested PCR method was used to obtain the expected 1,234 bp band (Fig. 1A). The PCR amplification products of pEGFP-N1 and XPD cDNA were digested using HindIII and SalI, respectively. After gel recovery, ligation, transformation and plasmid extraction, enzymatic digestion was performed. Due to the presence of the $B g l I I$ restriction site at the multiple cloning site of pEGFP-N1 and the 3 '-end of XPD, $B g l \mathrm{II}$ single digestion resulted in 4,737 and 1,200 bp products, as shown in Fig. 1B. The eukaryotic expression vector pcDNA3.1(+) was digested with HindIII and XhoI, while the PCR products were digested with HindIII and SalI. The digestion products were subsequently ligated using SalI and XhoI sites (per the principle of homologous enzymes) to obtain the pcDNA3.1(+)/XPD plasmid. Following identification with HindIII and $X b a \mathrm{I}$, the present results were consistent with the expectations (Fig. 1C). Additionally, the obtained sequencing results (Fig. 2) were consistent with the human XPD sequence reported on GenBank.

Recombinant plasmidtransfection into A375 cells. The negative control (PBS), pEGFP-N1 and pEGFP-N1/XPD recombinant plasmids were transfected into malignant melanoma A375 cells using Lipofectamine ${ }^{\circledR}$ 2000. GFP expression was observed 


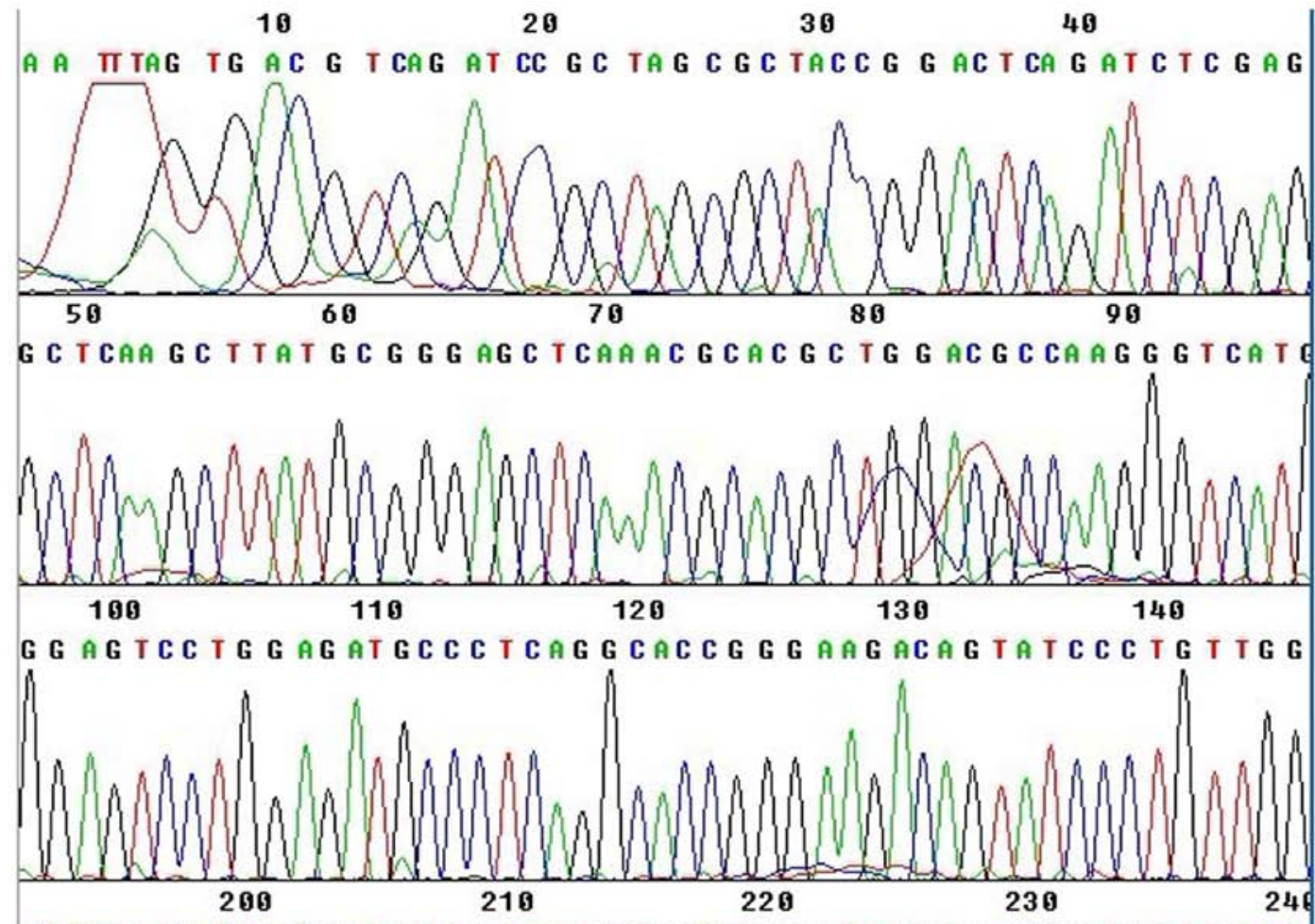

ACTCATCTACTGCTCAAG A ACTGTGCCAGAG ATTGAGAAGGTGATTGAA

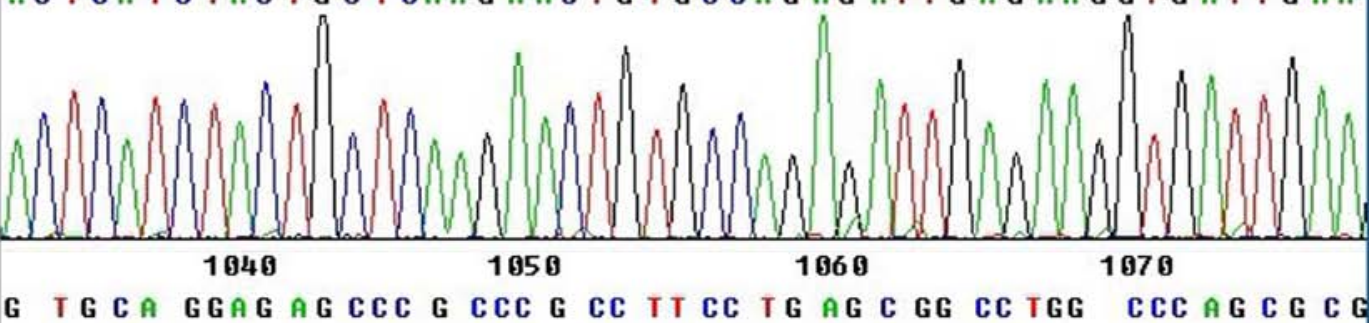

G TG CA GGAG AG CCC G CCC G CC TTCC TG AG C GG CC TGG CCC AGCG C
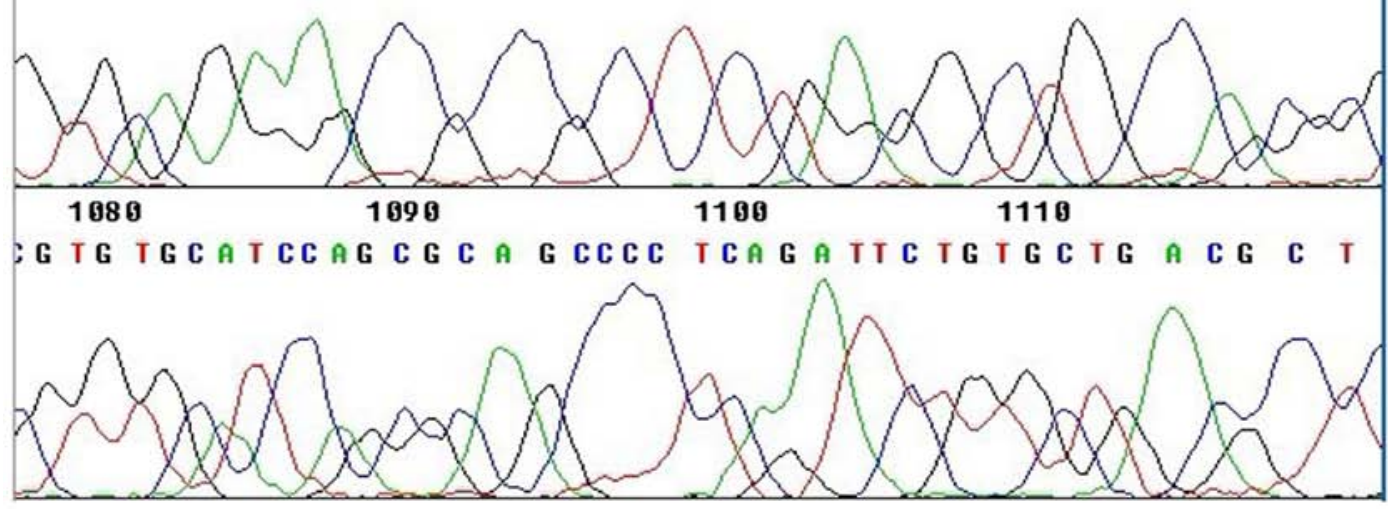

Figure 2. Recombinant plasmid sequencing results, consistent with the human xeroderma pigmentosum group D sequence on GenBank.

under a fluorescence microscope. The results revealed fluorescence in both the recombinant pEGFP-N1/XPD- and the empty pEGFP-N1 plasmid-transfected cells, while the negative control group exhibited no fluorescence, which proved that the recombinant plasmid was successfully transfected into A375 cells (Fig. 3).

Detection of XPD protein expression. Following transfection of pEGFP-N1/XPD and pcDNA3.1(+)/XPD into A375 cells, XPD expression was analyzed via western blot analysis. The results revealed that XPD was expressed in the transfection groups (Fig. 4). The $46 \mathrm{kDa}$ band corresponds to pcDNA3.1(+)/XPD, the $73 \mathrm{kDa}$ band corresponds to pEGFP-N1/XPD. As pEGFP-N1/XPD has green fluorescent protein, it can present as a $73 \mathrm{kDa}$ band which is larger than pcDNA3.1(+)/XPD.

Localization of XPD to the endoplasmic reticulum. Since pEGFP-N1/XPD -transfected A375 cells exhibited a clustered distribution of XPD-EGFP (as indicated by fluorescence microscopy), the subcellular location of XPD was analyzed. Using the Golgi membrane protein marker GM130 and the 


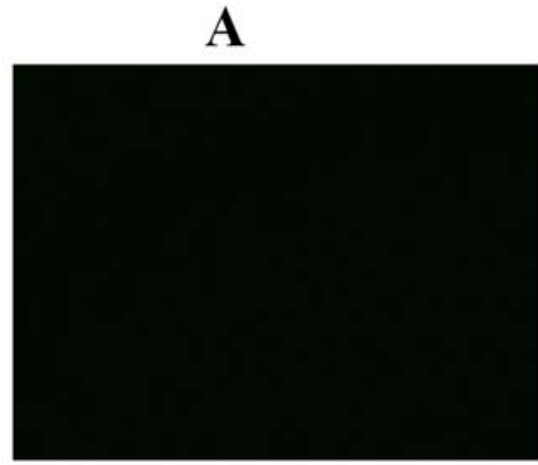

Negative control

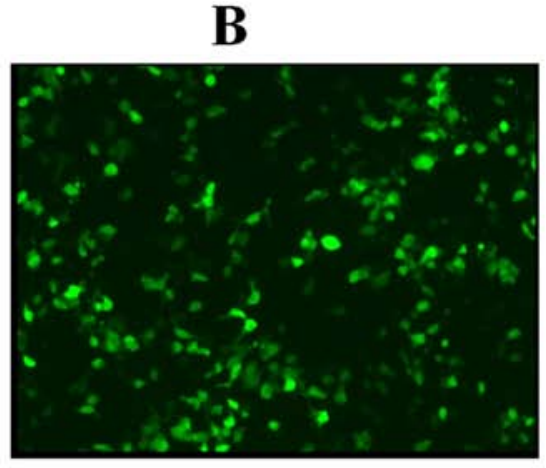

pEGFP-N1/XPD

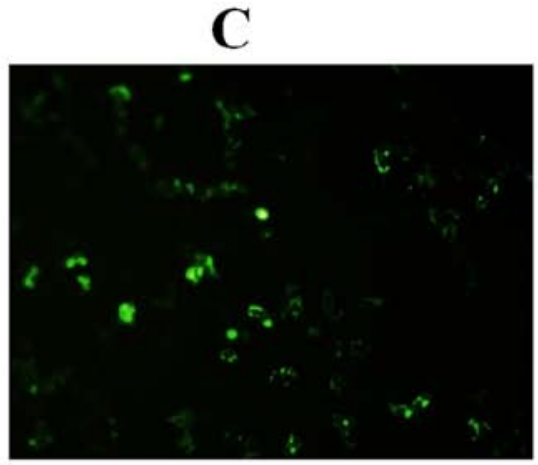

pcDNA3.1(+)/XPD

Figure 3. Recombinant plasmids transfected into A375 cells. GFP expression in A375 cells was observed under a fluorescence microscope (magnification, x200). (A) Negative control (A375 cells). (B) pEGFP-N1/XPD and (C) pcDNA3.1(+)/XPD transfected A375 cells. GFP, green fluorescent protein; XPD, xeroderma pigmentosum group D.

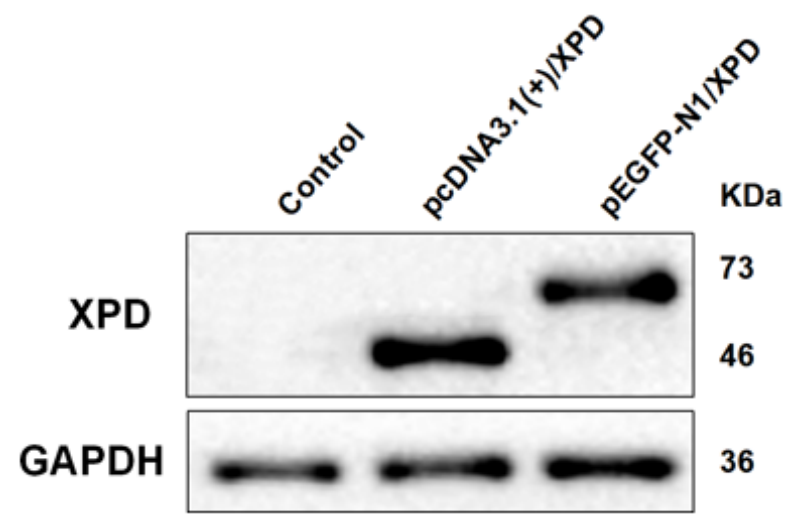

Figure 4. Detection of XPD protein expression in A375 cells transfected with pEGFP-N1/XPD and pcDNA3.1(+)/XPD. Western blot analysis Control consisted of A375 cells only. XPD, xeroderma pigmentosum group D.

endoplasmic reticulum membrane protein marker KDEL, immunofluorescence staining revealed that XPD was localized to the endoplasmic reticulum (Fig. 5).

Inhibition of A375 cell proliferation by XPD. Following transfection of pcDNA3.1(+) and pcDNA3.1(+)/XPD into A375 cells for 24,48 and $72 \mathrm{~h}$, cell proliferation was analyzed via MTT assay. The cell proliferation of the pcDNA3.1(+)/XPD group was significantly lower than that of the DMEM and pcDNA3.1(+) groups after 48 and $72 \mathrm{~h}$ of transfection $(\mathrm{P}<0.05$; Fig. 6), indicating that XPD inhibits the proliferation of A375 cells.

\section{Discussion}

In recent years, the incidence of skin cancer has been increasing worldwide, and this trend will continue to increase as the global population ages (10). This increase is primarily caused by the destruction of the ozone layer, which leads to an increase in the number of ultraviolet rays reaching the earth's surface (11). Previous study has demonstrated that UVB light can cause numerous types of DNA damage, leading to skin cancer (12). Therefore, DNA repair is important in maintaining the genetic integrity of the skin.
Normal human keratinocytes have a well-established DNA damage repair system to prevent gene mutations caused by UVB-associated DNA damage. The NER pathway is the primary means of repairing UVB-induced DNA damage, and the main line of defense against carcinogenesis caused by UVB rays (13). To date, two NER pathways have been discovered, including transcriptional coupling repair and whole genome nucleic acid excision repair, which are complex processes involving $>30$ gene products (14). XPD is the major protein of the nucleic acid excision repair process; the XPD gene is located on chromosome 19 and encodes a protein with ATP-dependent DNA helicase activity (7). The XPD protein is the second largest subunit of the TFIIH complex, which is composed of 9 subunits (namely XPB, XPD, p62,p52,p44,p34, cdk7, cyclinHT and MAT1); XPB, p62, p52, p44 and p34 form a core subcomplex object, while cdk7, cyclinHT and MAT1 form a subcomplex with the cdk active kinase (CAK) (3). XPD acts primarily as a scaffold that mediates CAK anchoring on the core subcomplex (5). During NER, XPD is responsible for opening the DNA duplex at the damaged position from the 5 ' to 3 'direction, allowing damage-specific nucleases to cut the damaged DNA from both sides (5). During transcription, the role of XPD is to maintain the structural stability of the TFIIH complex and to promote transcription amplification (6). Therefore, XPD serves an important role in the TFIIH complex-mediated NER and its transcription process (3). In addition, XPD participates in various physiological and pathological processes, such as cellular proliferation, apoptosis and tumorigenesis $(13,15-22)$. In the present study, the XPD gene was cloned into a eukaryotic expression vector to construct the pEGFP-N1/XPD recombinant plasmid; this was confirmed by sequencing, which was consistent with the XPD sequence published on GenBank.

Malignant melanoma is a type of malignant tumor originating from neural crest melanocytes (22). It mainly occurs in the skin and is the third most common skin malignant tumor (22). Additionally, it commonly results in metastasis and relapse, leading to a poor prognosis (11). The etiology of malignant melanoma is not fully understood, but an animal study has confirmed that UVB is closely associated with malignant melanoma formation (23). Indeed, UVB can cause various types of DNA damage, and can lead 
A
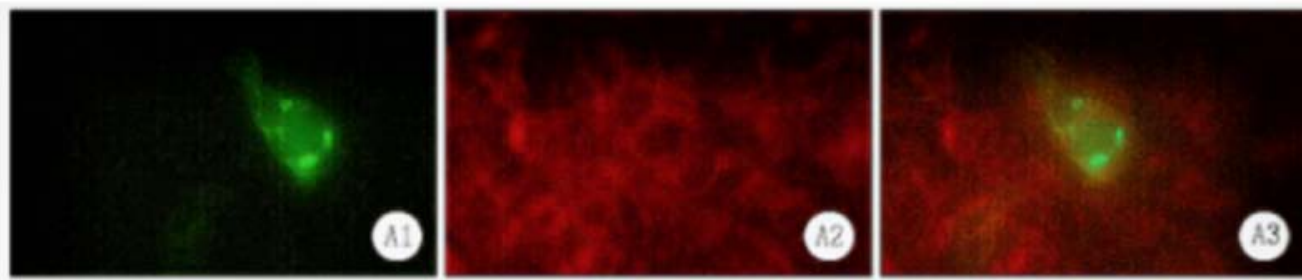

B
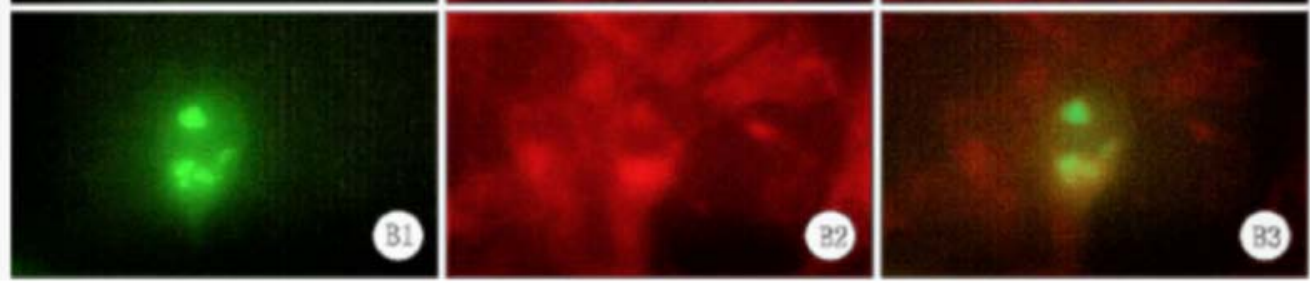

Figure 5. XPD is localized in the endoplasmic reticulum. (A) Immunofluorescence detection of XPD and the Golgi marker GM130, suggesting that there is no co-localization (A1, XPD; A2, GM130; A3, merge). (B) Immunofluorescence detection of XPD and the endoplasmic reticulum marker KDEL, suggesting that these 2 entities are colocalized coexist (B1, XPD; B2, KDEL; B3, merge). XPD, xeroderma pigmentosum group D.

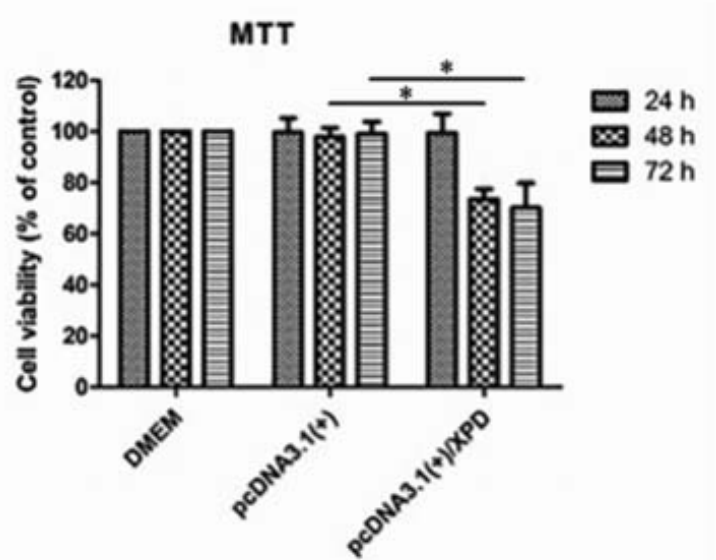

Figure 6. XPD inhibits the proliferation of A375 cells. Cellular proliferation was detected by MTT assay after transfecting A375 cells with pcDNA3.1(+) and pcDNA3.1(+)/XPD for 24,48 and $72 \mathrm{~h}$. Data are represented as the mean $\pm \mathrm{SD}(n=3)$. ANOVA was used to determine significant differences among the DMEM, pcDNA3.1(+) and pcDNA3.1(+)/XPD groups. " $\mathrm{P}<0.05$ compared with the control DMEM and pcDNA3.1(+) groups. XPD, xeroderma pigmentosum group $\mathrm{D}$.

to the development of cutaneous malignant melanoma (13). Studies on the molecular mechanisms of the susceptibility to cutaneous malignant melanoma genes have suggested that this susceptibility is associated with sequence variation mutations in NER genes $(2,24)$. Mutated XPD may be a biomarker of skin malignant melanoma (1). Kertat et al (25) revealed that the Gln/Gln genotype of the XPD 751 codon is a genetic marker of male malignant melanoma, and that the Lys/Gln genotype is important for predicting tumor progression.

To investigate the biological effects of XPD on malignant melanoma cells, the XPD gene was cloned and the recombinant $\mathrm{pEGFP-N1/XPD}$ plasmid was constructed and transfected into malignant melanoma A375 cells. GFP expression was confirmed by fluorescence expression of XPD-EGFP. Additionally, western blot analysis revealed that XPD was successfully expressed in transfected cells, indicating that an XPD eukaryotic expression system was successfully constructed. Furthermore, immunofluorescence analysis was used to detect the location of XPD in the endoplasmic reticulum. An MTT assay revealed that the proliferative capacity of malignant melanoma A375 cells transfected with pcDNA3.1(+)/XPD was significantly lower than that of cells transfected with pcDNA3.1(+) or DMEM, suggesting that XPD inhibits the proliferation and metabolism of A375 cells. Therefore, the present study provides a basis to further clarify the function of XPD in malignant melanoma.

\section{Acknowledgements}

Not applicable.

\section{Funding}

The present study was supported by the National Natural Science Foundation (grant no. 81350023).

\section{Availability of data and materials}

All data generated or analyzed during this study are included in this published article.

\section{Authors' contributions}

MG designed the study. YuW and YZ performed the experiments and wrote the manuscript. YaW and CP analyzed and interpreted data. All authors have read and approved the manuscript.

\section{Ethics approval and consent to participate}

Not applicable.

\section{Patient consent for publication}

Not applicable.

\section{Competing interests}

The authors declare that they have no competing interests. 


\section{References}

1. Lee TH and Kang TH: DNA oxidation and excision repair pathways. Int J Mol Sci 20: 6092, 2019.

2. Li C, Hu Z, Liu Z, Wang LE, Strom SS, Gershenwald JE, Lee JE, Ross MI, Mansfield PF, Cormier JN, et al: Polymorphisms in the DNA repair genes XPC, XPD, and XPG and risk of cutaneous melanoma: A case-control analysis. Cancer Epidemiol Biomarkers Prev 15: 2526-2532, 2006.

3. Lehmann AR: The xeroderma pigmentosum group D (XPD) gene: One gene, two functions, three diseases. Genes Dev 15: $15-23,2001$.

4. Tsao H: Update on familial cancer syndromes and the skin. J Am Acad Dermatol 42: 939-969, 2000.

5. Sobti RC, Berhane N, Melese D, Mahdi SA, Gupta L, Thakur H and Singh N: Impact of XPD gene polymorphism on risk of prostate cancer on North Indian population. Mol Cell Biochem 362: 263-268, 2012.

6. Schabath MB, Delclos GL, Grossman HB, Wang Y, Lerner SP, Chamberlain RM, Spitz MR and Wu X: Polymorphisms in XPD exons 10 and 23 and bladder cancer risk. Cancer Epidemiol Biomarkers Prev 14: 878-884, 2005.

7. Lovatt T, Alldersea J, Lear JT, Hoban PR, Ramachandran S, Fryer AA, Smith AG and Strange RC: Polymorphism in the nuclear excision repair gene ERCC2/XPD: Association between an exon 6-exon 10 haplotype and susceptibility to cutaneous basal cell carcinoma. Hum Mutat 25: 353-359, 2005.

8. Yin J, Li J, Ma Y, Guo L, Wang H and Vogel U: The DNA repair gene ERCC2/XPD polymorphism Arg 156Arg (A22541C) and risk of lung cancer in a Chinese population. Cancer Lett 223: 219-226, 2005.

9. https://www.uniprot.org/ niport/?query=001130867\&sort=score

10. Armstrong BK, Kricker A and English DR: Sun exposure and skin cancer. Australas J Dermatol 38 (Suppl 1): S1-S6, 1997.

11. Swetter SM, Johnson TM, Miller DR, Layton CJ, Brooks KR and Geller AC: Melanoma in middle aged older men: A multi-institutional survey study of factors related to tumor thickness. Arch Dermatol 145: 397-404, 2009.

12. Afaq F, Adhami VM and Mukhtar H: Photochemoprevention of ultraviolet B signaling and photocarcinogenesis. Mutat Res 571: 153-173, 2005.

13. Alisha A, Perween N, Saijo M, Ghaskadbi SS and Ghaskadbi S: Analysis of the conserved NER helicases (XPB and XPD) and UV-induced DNA damage in Hydra. Biochim Biophys Acta Gen Subj 1862: 2031-2042, 2018.

14. Wood RD, Mitchell M and Lindahl T: Human DNA repair genes, 2005. Mutat Res 577: 275-283, 2005.

15. Kuptsova-Clarkson N, Ambrosone CB, Weiss J, Baer MR, Sucheston LE, Zirpoli G, Kopecky KJ, Ford L, Blanco J, Wetzler M and Moysich KB: XPD DNA nucleotide excision repair gene polymorphisms associated with DNA repair deficiency predict better treatment outcomes in secondary acute myeloid leukemia. Int J Mol Epidemiol Genet 1: 278-294, 2010.
16. Wang HY, Xiong GF, Zhang JX, Xu H, Guo WH, Xu JJ and Xiong XY: The role of XPD in cell apoptosis and viability and its relationship with p53 and cdk2 in hepatoma cells. Med Oncol 29: 161-167, 2012.

17. Yilmaz E, Celik O, Celik E, Turkcuoglu I, Simsek Y, Karaer A, Otlu B, Gulbay G and Yesilada E: XPD and XRCC1 gene polymorphism in patients with normal and abnormal cervical cytology by pap smear. Eur Rev Med Pharmacol Sci 16: 1713-1718, 2012.

18. Szkandera J, Absenger G, Liegl-Atzwanger B, Pichler M, Stotz M, Gerger S, Zacherl M, Renner W, Haijun M, Leithner A and Gerger A: Common gene variants in RAD51, XRCC2 and XPD are not associated with clinical outcome in soft-tissue sarcoma patients. Cancer Epidemiol 37: 1003-1009, 2013.

19. Constantinescu-Aruxandei D, Petrovic-Stojanovska B, Penedo JC, White MF and Naismith JH: Mechanism of DNA loading by the DNA repair helicase XPD. Nucleic Acids Res 44: 2806-2815, 2016

20. Shkarupa VM, Mishcheniuk OY, Henyk-Berezovska SO, Palamarchuk VO and Klymenko SV: Polymorphism of DNA repair gene XPD Lys751Gln and chromosome aberrations in lymphocytes of thyroid cancer patients exposed to ionizing radiation due to the chornobyl accident. Exp Oncol 38: 257-260, 2016.

21. Santovito A, Delsoglio M, Manitta E, Picco G, Meschiati G, Chiarizio M, Gendusa C and Cervella P: Association of GSTT1 null, XPD 751 CC and XPC 939 CC genotypes with increased levels of genomic damage among hospital pathologists. Biomarkers 22: 557-565, 2017.

22. de Carvalho Lima EN, Piqueira JRC and Maria DA: Advances in carbon nanotubes for malignant melanoma: A chance for treatment. Mol Diagn Ther 22: 703-715, 2018.

23. Pfeifer GP and Besaratinia A: UV wavelength-dependent DNA damage and human non-melanoma and melanoma skin cancer. Photochem Photobiol Sci 11: 90-97, 2012.

24. Goode EL, Ulrich CM and Potter JD: Polymorphisms in DNA repair genes and associations with cancer risk. Cancer Epidemiol Biomarkers Prev 11: 1513-1530, 2002.

25. Kertat K, Rosdahl I, Sun XF, Synnerstad I and Zhang H: The Gln/Gln genotype of XPD codon 751 as a genetic marker for melanoma risk and Lys/Gln as an important predictor for melanoma progression: A case control study in the Swedish population. Oncol Rep 20: 179-183, 2008.

This work is licensed under a Creative Commons Attribution-NonCommercial-NoDerivatives 4.0 International (CC BY-NC-ND 4.0) License. 